\title{
Existence of positive solutions of a third order nonlinear differential equation with positive and negative terms
}

\section{Demou Luo* (D)}

\section{"Correspondence:}

scutldm@163.com

School of Applied Mathematics,

Guangdong University of

Technology, Guangzhou, P.R. China

\begin{abstract}
In this article we investigate the existence of positive solutions for a third order nonlinear differential equation with positive and negative terms. The main tool employed here is Kiguradze's lemma of classification of positive solutions. The asymptotic properties of solutions are also discussed. Two examples are also given to illustrate our result.
\end{abstract}

MSC: Primary 34A34; 34K13; secondary 34K30; 34L30

Keywords: Positive solutions; Asymptotic properties; Delay argument; Positive and negative term

\section{Introduction}

In 1993, Kiguradze and Chanturia [1] introduced the theory of asymptotic properties of solutions of nonautonomous ordinary differential equations as a method of continuum calculi. Since Kiguradze's groundbreaking work, there has been a significant growth in the theory of nonautonomous differential equations with deviating argument covering a variety of different problems; see [2-14] and the references therein.

Let $\mathbb{T}$ be a time scale such that $t_{0} \in \mathbb{T}$. In this article, we are interested in the analysis of qualitative theory of positive solutions of third order nonlinear differential equations. Motivated by the papers $[1,15]$ and the references therein, we consider the following dynamic equation:

$$
\left(b(t)\left(a(t) x^{\prime}(t)\right)^{\prime}\right)^{\prime}+p(t) f(x(\tau(t)))-q(t) g(x(\sigma(t)))=0, \quad t_{0} \leq t .
$$

Throughout this paper we assume that

$\left(H_{1}\right) a(t), b(t), p(t), q(t), \tau(t), \sigma(t) \in C\left(\left[t_{0}, \infty\right)\right)$ are positive;

$\left(H_{2}\right) f(u), h(u) \in C(\mathbb{R}), u f(u)>0, u h(u)>0$ for $u \neq 0, g$ is bounded, $f$ is nondecreasing;

$\left(H_{3}\right)-f(-u v) \geq f(u v) \geq f(u) f(v)$ for $u v>0$, and $f(u) \leq u$;

$\left(H_{4}\right) \tau(t) \leq t, \lim _{t \rightarrow \infty} \tau(t)=\infty, \lim _{t \rightarrow \infty} \sigma(t)=\infty$.

We consider the canonical case of (1.1), that is,

$\left(H_{5}\right) \int_{t_{0}}^{\infty} \frac{1}{a(s)} d s=\int_{t_{0}}^{\infty} \frac{1}{b(s)} d s=\infty$.

By a solution of Eq. (1.1), we can easily understand a function $x(t)$ with derivatives $a(t) x^{\prime}(t), b(t)\left(a(t) x^{\prime}(t)\right)^{\prime}$ continuous on $\left[T_{x}, \infty\right), T_{x} \geq t_{0}$, which satisfies Eq. (1.1) on $\left[T_{x}, \infty\right)$.

(c) The Author(s) 2018. This article is distributed under the terms of the Creative Commons Attribution 4.0 International License (http://creativecommons.org/licenses/by/4.0/), which permits unrestricted use, distribution, and reproduction in any medium, provided you give appropriate credit to the original author(s) and the source, provide a link to the Creative Commons license, and indicate if changes were made. 
We consider only those solutions $x(t)$ of (1.1) which satisfy $\sup \{|x(t)|: t \geq T\}>0$ for all $T \geq T_{x}$.

The research of the higher order ordinary differential equations (ODE) (see [1-7]) essentially takes advantage of some recapitulation of Kiguradze's lemma [1, 2]. In the lemma, from the fixed sign of the highest derivative, we can infer the form of possible nonoscillatory solutions. We cannot fix the sign of the fourth order quasi-derivative for an ultimately positive solution because the positive and negative terms are included in (1.1). So the authors primarily investigate the properties of (1.1) in the partial case when either $p(t) \equiv 0$ or $q(t) \equiv 0$.

In what follows we shall assume that $\left(H_{6}\right) \int_{t_{0}}^{\infty} \frac{1}{a(t)} \int_{t}^{\infty} \frac{1}{b(s)} \int_{s}^{\infty} q(u) d u d s d t<\infty$.

The organization of this paper is as follows. In Section 2, we introduce some definitions and lemmas and declare some preliminary material needed in later sections. We will state some facts about the differential equations with deviating argument as well as Kiguradze's lemma of classification of positive solutions. For details on Kiguradze's theorem, we refer the reader to [1]. In Section 3, we establish our main results for positive solutions by applying Kiguradze's classification of positive solutions theorem. In Section 4, we present the asymptotic properties of solutions. In Section 5, we give two examples to illustrate our results. The results presented in this paper extend the main results in [15].

\section{Preliminaries}

A time scale is an arbitrary nonempty closed subset of real numbers. The research of dynamic equations on time scales is an incredibly new area, and the number of studies on this subject is rapidly growing. The theory of dynamic equations unifies the theories of differential equations and difference equations. We suppose that the reader is familiar with the basic concepts concerning the calculus on time scales for dynamic equations. Otherwise one can find most of the material needed to read this paper in Kiguradze and Chanturia's books [1].

Definition 2.1 ([1]) A solution of (1.1) is termed oscillatory if it has arbitrarily large zeros on $\left[T_{x}, \infty\right)$, otherwise it is termed nonoscillatory. Eq. (1.1) is said to be oscillatory if all its solutions are oscillatory.

Definition 2.2 ([1]) The differential equation

$$
x^{(n)}(t)=p(t) x(t)
$$

has property $A$ if every solution of (2.1) for $n$ even is oscillatory and for $n$ odd either is oscillatory or satisfies the condition $\left|x^{(i)}(t)\right| \rightarrow 0$ as $t \rightarrow+\infty(i=1,2, \ldots, n-1)$.

Assume that (1.1) possesses an eventually positive solution $x(t)$ on $\left[T_{x}, \infty\right), T_{x} \geq t_{0}$. We introduce the auxiliary function $z(t)$ associated with $x(t)$ by

$$
z(t)=x(t)+\int_{t}^{\infty} \frac{1}{a(v)} \int_{v}^{\infty} \frac{1}{b(s)} \int_{s}^{\infty} q(u) g(x(\sigma(u))) d u d s d v
$$

It follows from $\left(H_{6}\right)$ and the boundedness of $h(u)$ that the definition of function $z(t)$ is correct and $z(t)$ exists for all $t \geq T_{x}$. It is useful to notice that $z(t)>x(t)>0, z^{\prime}(t)<x^{\prime}(t)$, 
and

$$
\left(b(t)\left(a(t) z^{\prime}(t)\right)^{\prime}\right)^{\prime}=-p(t) f(x(\tau(t)))<0 .
$$

Theorem 2.3 Assume that all the solutions of

$$
z^{\prime \prime}(t)+p(t) z(t)=0
$$

are nonoscillatory. Then every positive solution $z(t)$ of (1.1) satisfies either

$$
z(t) \in \mathcal{N}_{0} \quad \Longleftrightarrow \quad a(t) z^{\prime}(t)<0, \quad b(t)\left(a(t) z^{\prime}(t)\right)^{\prime}>0, \quad\left(b(t)\left(a(t) z^{\prime}(t)\right)^{\prime}\right)^{\prime}<0,
$$

or

$$
z(t) \in \mathcal{N}_{2} \quad \Longleftrightarrow \quad a(t) z^{\prime}(t)>0, \quad b(t)\left(a(t) z^{\prime}(t)\right)^{\prime}>0, \quad\left(b(t)\left(a(t) z^{\prime}(t)\right)^{\prime}\right)^{\prime}<0 .
$$

Corollary 2.4 ([1]) Assume that

$$
\liminf _{t \rightarrow \infty} t^{2} p(t)<\frac{2}{3 \sqrt{3}},
$$

then the set $\mathcal{N}$ of all positive solutions of (1.1) has the following decomposition:

$$
\mathcal{N}=\mathcal{N}_{0} \cup \mathcal{N}_{2}
$$

In this article, to ensure qualitative theory of the studied equation, some easily verifiable conditions must be established. To predigest our notation, we denote that

$$
B(t)=\int_{t_{1}}^{t} \frac{1}{b(s)} d s
$$

and

$$
A(t)=\int_{t_{1}}^{t} \frac{1}{a(s)} \int_{t_{1}}^{s} \frac{1}{b(u)} d u d s
$$

Definition $2.5([1])$ A solution $x(t)$ of (1.1) defined on an interval $\left[t_{1},+\infty\right) \subset\left[t_{0},+\infty\right)$ is said to be a Kneser solution if

$$
(-1)^{i} x^{(i)}(t) x(t) \geq 0
$$

for $t_{0} \leq t_{1}(i=0,1, \ldots, n-1)$.

\section{Existence of positive solutions}

In this section we shall investigate the existence of positive solutions for Eq. (1.1). The main result is in the following theorem. 
Theorem 3.1 Let, for all $t_{1}$ large enough,

$$
\int_{t_{1}}^{\infty} p(s) f(A(\tau(s))) d s=\infty
$$

Assume that

$$
\begin{aligned}
& \limsup _{t \rightarrow \infty}\left\{\frac{1}{B(\tau(t))} \int_{t_{1}}^{\tau(t)} p(s) f(A(\tau(s))) B(s) d s\right. \\
&\left.\quad+\int_{\tau(t)}^{t} p(s) f(A(\tau(s))) d s+f(B(\tau(t))) \int_{t}^{\infty} p(s) f\left(\frac{A(\tau(s))}{B(\tau(s))}\right) d s\right\} \\
&>\limsup _{u \rightarrow 0} \frac{u}{f(u)} .
\end{aligned}
$$

Then the positive solution class $\mathcal{N}_{2}=\varnothing$.

Proof Assume on the contrary that (1.1) possesses an eventually positive solution $z(t) \in$ $\mathcal{N}_{3}$. Using the fact that $b(t)\left(a(t) z^{\prime}(t)\right)^{\prime}$ is decreasing, we have

$$
\begin{aligned}
a(t) z^{\prime}(t) & \geq \int_{t_{1}}^{t} b(s)\left(a(s) z^{\prime}(s)\right)^{\prime} \frac{1}{b(s)} d s \geq b(t)\left(a(t) z^{\prime}(t)\right)^{\prime} \int_{t_{1}}^{t} \frac{1}{b(s)} d s \\
& =b(t)\left(a(t) z^{\prime}(t)\right)^{\prime} B(t) .
\end{aligned}
$$

In view of (3.3), we can see that $\left(\frac{a(t) z^{\prime}(t)}{B(t)}\right)^{\prime} \leq 0$; consequently, $\frac{a(t) z^{\prime}(t)}{B(t)}$ is decreasing. Then

$$
\begin{aligned}
x(t) & \geq \int_{t_{1}}^{t} x^{\prime}(s) d s \geq \int_{t_{1}}^{t} z^{\prime}(s) d s=\int_{t_{1}}^{t} \frac{a(s) z^{\prime}(s)}{B(s)} \frac{B(s)}{a(s)} d s \\
& \geq \frac{a(t) z^{\prime}(t)}{B(t)} \int_{t_{1}}^{t} \frac{B(s)}{a(s)} d s=\frac{a(t) z^{\prime}(t)}{B(t)} A(t) .
\end{aligned}
$$

Setting the last estimate into (2.3), we see that $y(t)=a(t) z^{\prime}(t)$ is a positive solution of the differential inequality

$$
\left(b(t) y^{\prime}(t)\right)^{\prime}+p(t) f\left(\frac{A(\tau(t))}{B(\tau(t))} y(\tau(t))\right) \leq 0 .
$$

What is more, $\frac{y(t)}{B(t)}$ is decreasing and $b(t) y^{\prime}(t)>0$.

On the other hand, an integration of (3.4) from $t$ to $\infty$ and then from $t_{1}$ to $t$ yields

$$
\begin{aligned}
y(t) \geq & \int_{t_{1}}^{t} \frac{1}{b(u)} \int_{u}^{\infty} p(s) f\left(\frac{A(\tau(s))}{B(\tau(s))} y(\tau(s))\right) d s d u \\
= & \int_{t_{1}}^{t} \frac{1}{b(u)} \int_{u}^{t} p(s) f\left(\frac{A(\tau(s))}{B(\tau(s))} y(\tau(s))\right) d s d u \\
& +\int_{t_{1}}^{t} \frac{1}{b(u)} \int_{t}^{\infty} p(s) f\left(\frac{A(\tau(s))}{B(\tau(s))} y(\tau(s))\right) d s d u
\end{aligned}
$$




$$
\begin{aligned}
= & \int_{t_{1}}^{t} p(s) f\left(\frac{A(\tau(s))}{B(\tau(s))} y(\tau(s))\right) B(s) d s \\
& +B(t) \int_{t}^{\infty} p(s) f\left(\frac{A(\tau(s))}{B(\tau(s))} y(\tau(s))\right) d s .
\end{aligned}
$$

Having replaced $t$ by $\tau(t)$ in the last inequality, we obtain

$$
\begin{aligned}
y(\tau(t)) \geq & \int_{t_{1}}^{\tau(t)} p(s) f\left(\frac{A(\tau(s))}{B(\tau(s))} y(\tau(s))\right) B(s) d s \\
& +B(\tau(t)) \int_{\tau(t)}^{t} p(s) f\left(\frac{A(\tau(s))}{B(\tau(s))} y(\tau(s))\right) d s \\
& +B(\tau(t)) \int_{t}^{\infty} p(s) f\left(\frac{A(\tau(s))}{B(\tau(s))} y(\tau(s))\right) d s .
\end{aligned}
$$

Employing $\left(H_{3}\right)$ and the fact that $y(t)$ is increasing and $\frac{y(t)}{B(t)}$ is decreasing, we have

$$
\begin{aligned}
y(\tau(t)) \geq & f\left(\frac{y(\tau(t))}{B(\tau(t))}\right) \int_{t_{1}}^{\tau(t)} p(s) f(A(\tau(s))) B(s) d s \\
& +B(\tau(t)) f\left(\frac{y(\tau(t))}{B(\tau(t))}\right) \int_{\tau(t)}^{t} p(s) f(A(\tau(s))) d s \\
& +B(\tau(t)) f(y(\tau(t))) \int_{t}^{\infty} p(s) f\left(\frac{A(\tau(s))}{B(\tau(s))}\right) d s .
\end{aligned}
$$

Therefore, setting $u=\frac{y(\tau(t))}{B(\tau(t))}$, we get

$$
\begin{aligned}
\frac{u}{f(u)} \geq & \frac{1}{B(\tau(t))} \int_{t_{1}}^{\tau(t)} p(s) f(A(\tau(s))) B(s) d s+\int_{\tau(t)}^{t} p(s) f(A(\tau(s))) d s \\
& +f(B(\tau(t))) \int_{t}^{\infty} p(s) f\left(\frac{A(\tau(s))}{B(\tau(s))}\right) d s .
\end{aligned}
$$

Condition (3.1) guarantees that $\frac{y(t)}{B(t)} \rightarrow 0$ as $t \rightarrow \infty$. Indeed, if we admit $\frac{y(t)}{B(t)} \rightarrow \ell>0$, then $\frac{y(t)}{B(t)} \geq \ell$ and setting the last inequality into (3.4), we obtain

$$
\left(b(t) y^{\prime}(t)\right)^{\prime}+p(t) f(\ell) f(A(\tau(t))) \leq\left(b(t) y^{\prime}(t)\right)^{\prime}+p(t) f(\ell A(\tau(t))) \leq 0 .
$$

An integration from $t_{1}$ to $\infty$ yields

$$
b\left(t_{1}\right) y^{\prime}\left(t_{1}\right) \geq f(\ell) \int_{t_{1}}^{\infty} p(s) f(A(\tau(s))) d s,
$$

which contradicts condition (3.1). Now, we can take lim sup on both sides of (3.6), one gets a contradiction to (3.2).

Obviously, we have the following easily verifiable criterion for some special cases of (1.1).

Corollary 3.2 Let, for all $t_{1}$ large enough,

$$
\int_{t_{1}}^{\infty} p(s) A(\tau(s)) d s=\infty
$$




$$
\begin{aligned}
& \limsup _{t \rightarrow \infty}\left\{\frac{1}{B(\tau(t))} \int_{t_{1}}^{\tau(t)} p(s) A(\tau(s)) B(s) d s+\int_{\tau(t)}^{t} p(s) A(\tau(s)) d s\right. \\
& \left.\quad+B(\tau(t)) \int_{t}^{\infty} p(s) \frac{A(\tau(s))}{B(\tau(s))} d s\right\} \geq 1 .
\end{aligned}
$$

Then the positive solution class $\mathcal{N}_{2}$ of third order trinomial differential equation

$$
\left(b(t)\left(a(t) x^{\prime}(t)\right)^{\prime}\right)^{\prime}+p(t) x(\tau(t))-q(t) g(x(\sigma(t)))=0
$$

is empty.

Theorem 3.3 Let, for all $t_{1}$ large enough,

$$
\int_{t_{1}}^{\infty} \frac{1}{b(u)} \int_{u}^{\infty} p(s) f\left(\frac{A(\tau(s))}{B(\tau(s))}\right) d s d u=\infty
$$

Assume that

$$
\begin{aligned}
\limsup _{t \rightarrow \infty}\left\{f\left(\frac{1}{B(\tau(t))}\right) \int_{t_{1}}^{\tau(t)} p(s) f(A(\tau(s))) B(s) d s\right. \\
+B(\tau(t)) f\left(\frac{1}{B(\tau(t))}\right) \int_{\tau(t)}^{t} p(s) f(A(\tau(s))) d s \\
\left.+B(\tau(t)) \int_{t}^{\infty} p(s) f\left(\frac{A(\tau(s))}{B(\tau(s))}\right) d s\right\} \\
>\limsup _{u \rightarrow \infty} \frac{u}{f(u)}
\end{aligned}
$$

Then the positive solution class $\mathcal{N}_{2}$ of (1.1) is empty.

Proof Assume that $x(t)$ is a positive solution of (1.1). Proceeding exactly as in the proof of Theorem 3.1, we verify that the associated function $z(t)$ belongs to the situation of $z(t) \in$ $\mathcal{N}_{2}$.

If $z(t) \in \mathcal{N}_{2}$, then $y(t)=a(t) z^{\prime}(t)$ satisfies (3.5). We claim that condition (3.10) implies that $y(t) \rightarrow \infty$ as $t \rightarrow \infty$. Really, if not, then $y(t) \rightarrow L$ as $t \rightarrow \infty$. An integration of (3.4) from $t$ to $\infty$ yields

$$
b(t) y^{\prime}(t) \geq \int_{t}^{\infty} p(s) f\left(\frac{A(\tau(s))}{B(\tau(s))} y(\tau(s))\right) d s .
$$

Integrating once more, we get

$$
\begin{aligned}
y(t) & \geq \int_{t_{1}}^{\infty} \frac{1}{b(u)} \int_{u}^{\infty} p(s) f\left(\frac{A(\tau(s))}{B(\tau(s))} y(\tau(s))\right) d s d u \\
& \geq f\left(y\left(\tau\left(t_{1}\right)\right)\right) \int_{t_{1}}^{\infty} \frac{1}{b(u)} \int_{u}^{\infty} p(s) f\left(\frac{A(\tau(s))}{B(\tau(s))}\right) d s d u .
\end{aligned}
$$

This is in contradiction to (3.10), and we conclude that $y(t) \rightarrow \infty$ as $t \rightarrow \infty$. 
Thus, we can set that $u=y(\tau(t))$, and we obtain

$$
\begin{aligned}
\frac{u}{f(u)} \geq & f\left(\frac{1}{B(\tau(t))}\right) \int_{t_{1}}^{\tau(t)} p(s) f(A(\tau(s))) B(s) d s \\
& +B(\tau(t)) f\left(\frac{1}{B(\tau(t))}\right) \int_{\tau(t)}^{t} p(s) f(A(\tau(s))) d s \\
& +B(\tau(t)) \int_{t}^{\infty} p(s) f\left(\frac{A(\tau(s))}{B(\tau(s))}\right) d s .
\end{aligned}
$$

Now, we can take limsup on both sides of (3.12), one gets a contradiction to (3.11). The proof is complete now.

Theorem 3.4 Let, for all t $t_{1}$ large enough,

$$
\int_{t_{1}}^{\infty} \frac{1}{a(v)} \int_{v}^{\infty} \frac{1}{b(s)} \int_{s}^{\infty} p(u) d u d s d v=\infty
$$

Then the positive solution class $\mathcal{N}_{0}=\varnothing$.

Proof Assume on the contrary that (1.1) possesses an eventually positive solution $z(t) \in$ $\mathcal{N}_{0}$. Let us denote that $Z^{\prime}(t)=z(t)$ and $Z(t)>0$. Due to $z(t)>0$ and $z^{\prime}(t)<0$, then $\int_{t_{1}}^{t} z(s) d s \geq t z(t)-t_{1} z(t)$ and $Z\left(t_{1}\right)-t_{1} z(t) \geq 0$, we obtain

$$
Z(t)=Z\left(t_{1}\right)+\int_{t_{1}}^{t} z(s) d s \geq t z(t)+Z\left(t_{1}\right)-t_{1} z(t) \geq t z(t)
$$

Using the fact that $u f(u)>0$ and $f$ is nondecreasing, we have $f(x(\tau(t))) \geq \varepsilon>0$. An integration of (2.3) from $t$ to $\infty$ yields

$$
b(t)\left(a(t) z^{\prime}(t)\right)^{\prime} \geq \int_{t}^{\infty} p(s) f(x(\tau(s))) d s \geq \varepsilon \int_{t}^{\infty} p(s) d s .
$$

Integrating from $t$ to $\infty$ and consequently from $t_{1}$ to $\infty$, we obtain

$$
z\left(t_{1}\right) \geq \varepsilon \int_{t_{1}}^{\infty} \frac{1}{a(v)} \int_{v}^{\infty} \frac{1}{b(s)} \int_{s}^{\infty} p(u) d u d s d v
$$

We get a contradiction to (3.13), and the proof is complete.

Theorem 3.5 Let (2.5) hold. Assume that all the conditions of Theorems 3.1 and 3.4 hold. Then Eq. (1.1) has no positive solutions.

Theorem 3.6 Let (2.5) hold. Assume that all the conditions of Theorems 3.3 and 3.4 hold. Then Eq. (1.1) has no positive solutions.

Theorem 3.7 Let (2.5) hold. Assume that all the conditions of Corollary 3.2 and Theorem 3.4 hold. Then Eq. (3.9) has no positive solutions. 


\section{Asymptotic properties}

In this section some asymptotic properties of positive solutions of (1.1) are treated. Firstly, we need to transform (1.1). Let us denote that

$$
\begin{aligned}
& \mathcal{D}_{0}(t)=\frac{p(t) f(x(\tau(t)))-q(t) g(x(\sigma(t)))}{a(t) b(t)}, \\
& \mathcal{D}_{1}(t)=\frac{a^{\prime}(t) b^{\prime}(t)+a^{\prime \prime}(t) b(t)}{a(t) b(t)}, \\
& \mathcal{D}_{2}(t)=\frac{a(t) b^{\prime}(t)+2 a^{\prime}(t) b(t)}{a(t) b(t)},
\end{aligned}
$$

and

$$
\mathcal{F}\left(t, x(t), x^{\prime \prime}(t), x^{\prime \prime \prime}(t)\right)=-\left(\mathcal{D}_{2}(t) x^{\prime \prime}(t)+\mathcal{D}_{1}(t) x^{\prime}(t)+\mathcal{D}_{0}(t)\right) .
$$

Obviously, Eq. (1.1) will be transformed as follows:

$$
x^{\prime \prime \prime}(t)+\mathcal{D}_{2}(t) x^{\prime \prime}(t)+\mathcal{D}_{1}(t) x^{\prime}(t)+\mathcal{D}_{0}(t)=0 .
$$

Below we establish the existence criteria for Kneser solutions of (4.1) and study the asymptotics of these solutions.

Theorem 4.1 Let

$$
\mathcal{F}(t, 0,0,0)=\frac{q(t) g(0)-p(t) f(0)}{a(t) b(t)}=0
$$

for $t \geq t_{1}, m \in\{0,1\}, r \in(0,+\infty)$ and

$$
(-1)^{m+3} \mathcal{F}\left(t, x_{1}, x_{2}, x_{3}\right) \geq 0
$$

for $t \geq t_{1}, 0 \leq(-1)^{m+i-1} x_{i} \leq r t^{1-i}, i=1,2,3$. Then Eq. (4.1) has a continuum of Kneser solutions satisfying the conditions

$$
(-1)^{m+i} x^{(i)}(t) \geq 0
$$

for $t \geq t_{1}, i=0,1,2$.

Proof Set

$$
\mathcal{F}^{*}(t)=\max \left\{\left|\mathcal{F}\left(t, x_{1}, x_{2}, x_{3}\right)\right|: 0 \leq(-1)^{m+i-1} x_{i} \leq r t^{1-i}\right\}, \quad i=1,2,3,
$$

and choose $\delta \in\left(0, \frac{1}{t_{1}+1}\right)$ so small that

$$
2\left(t_{1}+1\right)^{2} \int_{t_{1}}^{t_{1}+\delta} \mathcal{F}^{*}(\tau) d \tau \leq \tau
$$


To prove the theorem, it suffices to show that if $r_{0}=\left(\frac{\delta}{t_{1}+\delta}\right)^{2} \frac{r}{12}$, then for any $c_{0} \in\left[0, r_{0}\right]$ Eq. (4.1) together with the condition

$$
x\left(t_{1}\right)=(-1)^{m} c_{0} .
$$

Consider the functions

$$
\chi_{i}(t, x)= \begin{cases}0, & (-1)^{m+i-1} x<0 \\ x, & 0 \leq(-1)^{m+i-1} x \leq r t^{1-i} \\ r t^{1-i}, & (-1)^{m+i-1} x>r t^{1-i}\end{cases}
$$

and $\widetilde{\mathcal{F}}\left(t, x_{1}, x_{2}, x_{3}\right)=\mathcal{F}\left(t, \chi_{1}\left(t, x_{1}\right), \chi_{2}\left(t, x_{2}\right), \chi_{3}\left(t, x_{3}\right)\right)$. Obviously,

$$
\tilde{\mathcal{F}}\left(t, x_{1}, x_{2}, x_{3}\right)=\mathcal{F}\left(t, x_{1}, x_{2}, x_{3}\right)
$$

for $t \geq t_{1}, 0 \leq(-1)^{m+i-1} x_{i} \leq r t^{1-i}, i=1,2,3$. And

$$
\left|\tilde{\mathcal{F}}\left(t, x_{1}, x_{2}, x_{3}\right)\right| \leq \mathcal{F}^{*}(t)
$$

for $t \geq t_{1},\left(x_{1}, x_{2}, x_{3}\right) \in \mathbb{R}^{3}$.

On the other hand, (4.2) and (4.3) yield

$$
\widetilde{\mathcal{F}}\left(t, x_{1}, x_{2}, x_{3}\right)=0
$$

for $t \geq t_{1},(-1)^{m+i-1} x_{i} \leq 0, i=1,2,3$. And

$$
(-1)^{m+3} \widetilde{\mathcal{F}}\left(t, x_{1}, x_{2}, x_{3}\right) \geq 0
$$

for $t \geq t_{1},(-1)^{m+i-1} x_{i} \geq 0, i=1,2,3$.

By Lemma 10.1 of [1], for any positive integer $k$, the differential equation

$$
x^{\prime \prime \prime}(t)=\tilde{\mathcal{F}}\left(t, x(t), x^{\prime}(t), x^{\prime \prime}(t)\right)
$$

has a solution $x_{k}(t)$ satisfying the boundary conditions

$$
x_{k}\left(t_{1}\right)=(-1)^{m} c_{0}, \quad x_{k}^{i-1}\left(t_{1}+k\right)=0, \quad i=1,2 .
$$

Assuming that $(-1)^{m+2} x_{k}^{\prime \prime}\left(t_{1}+k\right)<0$, from (4.9) we obtain

$$
x_{k}(t)=\frac{1}{2} x_{k}^{\prime \prime}\left(t_{1}+k\right)\left(t-t_{1}-k\right)^{2}
$$

for $t_{1} \leq t \leq t_{1}+k,(-1)^{m} x_{k}\left(t_{1}\right)<0$.

But this is impossible because $(-1)^{m} x_{k}\left(t_{1}\right)=c_{0} \geq 0$. So

$$
(-1)^{m+2} x_{k}^{\prime \prime}\left(t_{1}+k\right) \geq 0 .
$$


According to (4.10), (4.11), and (4.12),

$$
(-1)^{m+i} x_{k}^{(i)}(t) \geq 0
$$

for $t_{1} \leq t \leq t_{1}+k, i=0,1,2,3$,

$$
\sum_{i=0}^{2} \frac{x_{k}^{(i)}(t)}{i !}\left(t-t_{1}\right)^{i} \leq\left|x\left(t_{1}\right)\right| \leq r_{0}
$$

for $t_{1} \leq t \leq t_{1}+k$, and

$$
\left|x_{k}^{(i)}\left(t_{1}+\delta\right)\right| \leq i ! r_{0} \delta^{-i}, \quad i=0,1,2
$$

Applying (4.5), (4.7), and (4.15), we get

$$
\begin{aligned}
\left|x_{k}^{(i)}(t)\right| \leq & \sum_{j=i}^{2} \frac{\left|x_{k}^{(j)}\left(t_{1}+\delta\right)\right|}{(j-i) !}\left(t_{1}+\delta-t\right)^{j-i} \\
& +\frac{1}{(2-i) !} \int_{t}^{t_{1}+\delta}(\tau-t)^{4-i} \mathcal{F}^{*}(\tau) d \tau \\
\leq & 3 ! r_{0} \delta^{-i}+\int_{t_{1}}^{t_{1}+\delta} \mathcal{F}^{*}(\tau) d \tau \\
\leq & {\left[3 ! r_{0}\left(\frac{t_{1}+\delta}{\delta}\right)^{i}+\frac{\left(t_{1}+\delta\right)^{i}}{2\left(t_{1}+1\right)^{2}} \cdot r\right] t^{-i} } \\
\leq & r t^{-i}
\end{aligned}
$$

for $t_{1} \leq t \leq t_{1}+\delta, i=0,1,2$. On the other hand, (4.14) implies

$$
\left|x_{k}^{(i)}(t)\right| \leq r t^{-i}
$$

for $t_{1}+\delta \leq t \leq t_{1}+k, i=0,1,2$. Therefore,

$$
\left|x_{k}^{(i)}(t)\right| \leq r t^{-i}
$$

for $t_{1} \leq t \leq t_{1}+k, i=0,1,2$.

It follows from (4.7), (4.13), and (4.17), that for any positive integer $k$ the function $x_{k}(t)$ is a solution of Eq. (4.1) on the interval $\left[t_{1}, t_{1}+k\right]$. By Lemma 10.2 of [1], $\left\{x_{k}(t)\right\}_{k=1}^{+\infty}$ contains a subsequence $\left\{x_{k_{l}}(t)\right\}_{l=1}^{+\infty}$ such that $\left\{x_{k_{l}}(t)^{(i)}\right\}_{l=1}^{+\infty}, i=0,1,2$, converge uniformly on every finite subinterval of $\left[t_{1},+\infty\right)$, and $x(t)=\lim _{l \rightarrow+\infty}\left\{x_{k_{l}}(t)\right\}$ for $t \geq t_{1}$ is a solution of Eq. (4.1). In view of (4.11) and (4.13), $x(t)$ satisfies conditions (4.4) and (4.6).

The proof of Theorem 4.1 is complete.

\section{Examples}

Example 5.1 Consider the third order trinomial differential equation

$$
\left(t^{\frac{1}{3}}\left(t^{\frac{1}{2}} x^{\prime}(t)\right)^{\prime}\right)^{\prime}+\frac{p}{t^{\frac{13}{6}}} x(\lambda t)-\frac{q}{t^{3}} \arctan (x(\sigma(t)))=0
$$


with $p>0, q>0, \lambda \in(0,1)$. Now $f(x)=x$ is nondecreasing and $h(x)=\arctan (x)$ is bounded. We can easily calculate that

$$
\begin{aligned}
& \int_{t_{1}}^{\infty} p(s) f(A(\tau(s))) d s \\
& \quad=\int_{t_{1}}^{\infty} p(s) A(\tau(s)) d s \\
& \quad=\int_{t_{1}}^{\infty} \frac{p}{s^{\frac{13}{6}}} \int_{t_{1}}^{\lambda s} \frac{1}{v^{\frac{1}{2}}} \int_{t_{1}}^{v} \frac{1}{u^{\frac{1}{3}}} d u d v d s \\
& =\infty .
\end{aligned}
$$

Then conditions (3.1) and (3.7) hold true. Due to

$$
\begin{aligned}
& \int_{t_{1}}^{\infty} \frac{1}{a(v)} \int_{v}^{\infty} \frac{1}{b(s)} \int_{s}^{\infty} p(u) d u d s d v \\
& \quad=\int_{t_{1}}^{\infty} \frac{1}{v^{\frac{1}{2}}} \int_{v}^{\infty} \frac{1}{s^{\frac{1}{3}}} \int_{s}^{\infty} \frac{p}{u^{\frac{13}{6}}} d u d s d v \\
& \quad=\infty
\end{aligned}
$$

condition (3.13) holds true. Therefore, the positive solution class $\mathcal{N}_{0}=\varnothing$. Simple computation shows that

$$
A(t) \sim \frac{9}{7} t^{\frac{7}{6}}, \quad B(t) \sim \frac{3}{2} t^{\frac{2}{3}}
$$

and (3.2), (3.10) take the form

$$
p \lambda^{\frac{7}{6}}\left(\frac{9}{7} \ln \left(\frac{1}{\lambda}\right)+\frac{27}{7}\right)>1
$$

and

$$
\begin{aligned}
& \int_{t_{1}}^{\infty} \frac{1}{b(u)} \int_{u}^{\infty} p(s) f\left(\frac{A(\tau(s))}{B(\tau(s))}\right) d s d u \\
& \quad=\int_{t_{1}}^{\infty} \frac{1}{u^{\frac{1}{3}}} \int_{u}^{\infty} \frac{p}{s^{\frac{13}{6}}}\left(\frac{\frac{9}{7} s^{\frac{7}{6}}}{\frac{3}{2} s^{\frac{2}{3}}}\right) d s d u \\
& \quad=\frac{6 p}{7} \int_{t_{1}}^{\infty} \frac{1}{u^{\frac{1}{3}}} \int_{u}^{\infty} \frac{1}{s^{\frac{5}{3}}} d s d u \\
& =\infty
\end{aligned}
$$

Therefore, the positive solution class $\mathcal{N}_{2}=\varnothing$. We can conclude that Eq. (5.1) has no positive solutions.

Our results are also applicable for the case when $\tau(t) \equiv t$.

Example 5.2 Consider the third order differential equation

$$
\left(t^{\frac{1}{3}}\left(t^{\frac{1}{2}} x^{\prime}(t)\right)^{\prime}\right)^{\prime}+\frac{p}{t^{\frac{13}{6}}} x(t)-\frac{q}{t^{3}} \arctan (x(\sigma(t)))=0
$$


with $p>0, q>0$. We can easily calculate that

$$
\begin{aligned}
& \int_{t_{1}}^{\infty} p(s) f(A(\tau(s))) d s \\
& \quad=\int_{t_{1}}^{\infty} p(s) A(\tau(s)) d s \\
& \quad=\int_{t_{1}}^{\infty} \frac{p}{s^{\frac{13}{6}}} \int_{t_{1}}^{s} \frac{1}{v^{\frac{1}{2}}} \int_{t_{1}}^{v} \frac{1}{u^{\frac{1}{3}}} d u d v d s \\
& =\infty .
\end{aligned}
$$

The following steps are the same as in Example 5.1, thus we omit them.

\section{Acknowledgements}

This work was supported in part by the National Natural Science Foundation of China under Grant No. 61673121, in part by the Natural Science Foundation of Guangdong Province under Grant No. 2014A030313507, and in part by the Projects of Science and Technology of Guangzhou under Grant No. 201508010008. The authors are grateful to the referee for careful reading of the paper and for his or her useful comments which helped them to improve the paper.

\section{Competing interests}

The authors declare that they have no competing interests.

\section{Authors' contributions}

All the authors contributed equally and significantly in writing this paper. All the authors read and approved the final manuscript.

\section{Publisher's Note}

Springer Nature remains neutral with regard to jurisdictional claims in published maps and institutional affiliations.

Received: 28 November 2017 Accepted: 7 February 2018 Published online: 12 March 2018

\section{References}

1. Kiguradze, I.T., Chanturia, T.A.: Asymptotic Properties of Solutions of Nonautonomous Ordinary Differential Equations. Kluwer Academic, Dordrecht (1993)

2. Kiguradze, I.T.: On the oscillatory character of solutions of the equation $\frac{d^{m} u}{d t^{m}}+a(t)|u|^{n} \operatorname{sign} u=0$. Mat. Sb. 65, 172-187 (1964) (in Russian)

3. Agarwal, R.P., Grace, S.R., O'Regan, D.: Oscillation Theory for Difference and Functional Differential Equations. Kluwer Academic, Dordrecht (2000)

4. Kitamura, Y.: Characterization of oscillation of fourth order functional differential equations with deviating arguments. Ann. Mat. Pura Appl. 124(1), 345-365 (1980)

5. Li, T., Baculíková, B., Džurina, J., Zhang, C.: Oscillation of fourth-order neutral differential equations with $p$-Laplacian like operators. Bound. Value Probl. 2014, Article ID 56 (2014)

6. Ruyun, M., Haiyan, W.: On the existence of positive solutions of fourth-order ordinary differential equations. Appl. Anal. 59(1-4), 225-231 (1995)

7. Ladde, G.S., Lakshmikantham, V., Zhang, B.G.: Oscillation Theory of Differential Equations with Deviating Arguments. Marcel Dekker, New York (1987)

8. Elsgolts, L.E., Norkin, S.B.: Introduction to the Theory and Application of Differential Equations with Deviating Arguments. Elsevier, Amsterdam (1973)

9. Bartušek, M., Došlá, Z.: Asymptotic problems for fourth-order nonlinear differential equations. Bound. Value Probl. 2013, Article ID 89 (2013)

10. Hou, C., Cheng, S.S.: Asymptotic dichotomy in a class of fourth-order nonlinear delay differential equations with damping. Abstr. Appl. Anal. 2009, Article ID 484158 (2009)

11. Agarwal, R., Grace, S.R., Manojlovic, J.V.: Oscillation criteria for certain fourth order nonlinear functional differential equations. Math. Comput. Model. 44(1), 163-187 (2006)

12. Liang, H.: Asymptotic behavior of solutions to higher order nonlinear delay differential equations. Electron. J. Differ. Equ. 2014, Article ID 186 (2014)

13. Swanson, C.H.A.: Comparison and Oscillation Theory of Linear Differential Equations. Elsevier, Amsterdam (1968)

14. Elias, U.: Oscillation Theory of Two-Term Differential Equations. Mathematics and Its Applications, vol. 396. Springer, Dordrecht (2013)

15. Džurina, J., Baculíková, B.: Property A of differential equations with positive and negative term. Electron. J. Qual. Theory Differ. Equ. 2017, Article ID 27 (2017) 